ANNALES

POLONICI MATHEMATICI

$83.2(2004)$

\title{
The theorem of Forelli for holomorphic mappings into complex spaces
}

\author{
by Pham Ngoc Mai, Do Duc Thai and Le Tai Thu (Hanoi)
}

\author{
Dedicated to Professor Nguyen Thanh Van \\ on the occasion of his 60th birthday
}

\begin{abstract}
Generalizations of the theorem of Forelli to holomorphic mappings into complex spaces are given.
\end{abstract}

1. Introduction. The classical Hartogs theorem states that if a complex-valued function $f\left(z_{1}, \ldots, z_{n}\right)$ defined for $z=\left(z_{1}, \ldots, z_{n}\right) \in U \subset \mathbb{C}^{n}$ $(n \geq 2)$ is separately holomorphic, i.e. holomorphic with respect to each variable separately when the other variables are fixed, then $f$ is jointly holomorphic. Equivalently, if $f$ is holomorphic on each line which is parallel to some coordinate axis, then $f$ is jointly holomorphic. Much attention has been given to generalizing this theorem, and many Hartogs-type theorems for separately holomorphic mappings have been obtained by various authors (see [Te], [S], [NZ1], [NZ2], [Shi2], [TM], [JP]).

Modifying the point of view of the above-mentioned theorem, in 1978, F. Forelli proved the following remarkable result (see $[\mathrm{Ru}, \mathrm{p} .60]$ or [Sha, p. 49]). If $f$ is a function defined in the unit ball $\mathbb{B}^{n} \subset \mathbb{C}^{n}$, holomorphic on the intersection of $\mathbb{B}^{n}$ with every complex line $l$ passing through the origin, and if $f$ is of class $\mathcal{C}^{\infty}$ in a neighbourhood of the origin, then it is holomorphic in $\mathbb{B}^{n}$.

Our main aim in this article is to generalize the theorem of Forelli to holomorphic mappings into complex spaces. Namely, we are going to prove the following:

Theorem A. Let $M$ be a complex space and $\mathbb{B}^{n}$ the open unit ball of $\mathbb{C}^{n}$. Let $f: \mathbb{B}^{n} \rightarrow M$ be a mapping such that $f$ is holomorphic on the intersection of $\mathbb{B}^{n}$ with every complex line $l$ passing through the origin, and $f$ is of

2000 Mathematics Subject Classification: 32A10, 32C10, 32H20, 32A17.

Key words and phrases: complex space of Hartogs type, Forelli property. 
class $\mathcal{C}^{\infty}$ in a neighbourhood of the origin. Then there exists a pluripolar subset $S$ of $\mathbb{P}^{n-1}(\mathbb{C})$ such that $f$ is holomorphic in a neighbourhood of $\mathbb{B}^{n}$ $\bigcup\{l: l \in S\}$.

TheOREm B. Let $M$ be a complex space of Hartogs type. Then $M$ has the Forelli property.

Theorem C. Let $M$ be a holomorphically convex Kähler complex space. Then $M$ has the Hartogs extension property if and only if $M$ has the Forelli property.

TheOREM D. Let $M$ be a holomorphically convex compact Kähler manifold. Let $f: \mathbb{B}^{n} \rightarrow M$ be a mapping such that $f$ is holomorphic on the intersection of $\mathbb{B}^{n}$ with every complex line l passing through the origin, and $f$ is of class $\mathcal{C}^{\infty}$ in a neighbourhood of the origin. Then $f$ is meromorphic in $\mathbb{B}^{n}$.

\section{Preliminaries}

2.1. Definition. For $r>0$ put $\Delta_{r}=\Delta(0, r)=\{|z|<r\} \subset \mathbb{C}$ and $\Delta_{1}=\Delta$.

Let $X$ be a complex space. We say that $X$ has the Hartogs extension property (briefly $X$ has (HEP)) if every holomorphic mapping, from a Riemann domain $\Omega$ over a Stein manifold into $X$, can be extended holomorphically to $\widehat{\Omega}$, the envelope of holomorphy of $\Omega$.

Let $H_{2}(r)=\left\{\left(z_{1}, z_{2}\right) \in \Delta^{2}:\left|z_{1}\right|<r\right.$ or $\left.\left|z_{2}\right|>1-r\right\}(0<r<1)$ denote the 2-dimensional Hartogs domain.

It is well known ([Shi1] or [I]) that $X$ has (HEP) iff every holomorphic mapping $f: H_{2}(r) \rightarrow X$ extends holomorphically over $\Delta^{2}$.

The class of complex spaces having (HEP) is large: it contains the taut complex spaces $[\mathrm{Fu}]$, complex Lie groups [ASY], and complete hermitian complex manifolds with non-positive holomorphic sectional curvature [Shi1]. In particular, Ivashkovich [I] showed that a holomorphically convex Kähler manifold has (HEP) iff it contains no rational curves. This was generalized to holomorphically convex Kähler spaces by Do Duc Thai [T].

2.2. Definition. Let $M$ be a complex space.

(i) An open subset $A$ of $M$ is said to be of type $(\mathrm{H})$ if there exists a biholomorphic mapping from $A$ onto an analytic subset of a complex space having (HEP).

(ii) The space $M$ is said to be of Hartogs type if for each $p \in M$ there exists a neighbourhood $W_{p}$ of $p$ and $r_{p}>0$ and a neighbourhood $S_{p}$ of $p$ of type (H) such that for each $f \in \operatorname{Hol}(\Delta, M)$, if $f(0) \in W_{p}$ then $f\left(\Delta_{r_{p}}\right) \subset S_{p}$. 
The class of complex spaces of Hartogs type is rather large. It is easy to see that it contains the complex spaces having (HEP) and the hyperbolic complex spaces.

2.3. Definition. Let $M$ be a complex space. We say that $M$ has the Forelli property for the unit ball $\mathbb{B}^{n}$ of $\mathbb{C}^{n}$ (briefly $M$ has (FP)) if whenever a mapping $f: \mathbb{B}^{n} \rightarrow M$ is holomorphic on the intersection of $\mathbb{B}^{n}$ with every complex line $l$ passing through the origin, and $f$ is of class $\mathcal{C}^{\infty}$ in a neighbourhood of the origin, then $f$ is holomorphic in $\mathbb{B}^{n}$.

Examples. (a) The complex plane $\mathbb{C}$ has the Forelli property (see $[\mathrm{Ru}$, p. 60]).

(b) Every complex space of Stein type has the Forelli property (see [TP]).

2.4. Let $l_{a}$ be a complex line passing through the origin of $\mathbb{C}^{n}$. Then in $\mathbb{C}^{n}$, the set $l_{a}$ is given by $\left\{t\left(a_{1}, \ldots, a_{n}\right): t \in \mathbb{C}\right\}$. Thus we can consider $l_{a}$ as a point $a=\left[a_{1}: \ldots: a_{n}\right]$ in $\mathbb{P}^{n-1}(\mathbb{C})$.

2.5. Let $S$ be a subset of a complex manifold $M$. We say that $S$ is pluripolar if for any $x_{0} \in S$ there are an open neighbourhood $U$ of $x_{0}$ in $M$ and a plurisubharmonic function $\varphi: U \rightarrow[-\infty, \infty)$ such that $S \cap U \subset$ $\{\varphi=-\infty\}$.

2.6. For $z=\left(z_{1}, \ldots, z_{n}\right) \in \mathbb{C}^{n}$, we let $\|z\|=\left(\left|z_{1}\right|^{2}+\cdots+\left|z_{n}\right|^{2}\right)^{1 / 2}$.

For each $R>0$ put $\mathbb{B}_{R}^{n}=\mathbb{B}^{n}(0, R)=\left\{z \in \mathbb{C}^{n}:\|z\|<R\right\}, \mathbb{B}^{n}=\mathbb{B}_{1}^{n}$.

3. Proofs of the main results. In order to prove Theorem A we need the following lemma:

3.1. Lemma ([Shi2]). Let $M$ be a complex space. Let $U, V$ be open sets in $\mathbb{C}^{m}, \mathbb{C}^{n}$ respectively and let $K$ be a connected compact set in $\mathbb{C}^{n}$ containing $V$. Let $f: U \times V \rightarrow M$ be a holomorphic map. If $f_{z}$ extends holomorphically to $K$ for all $z \in U$, then there exists a closed pluripolar subset $E$ of $U$ and a holomorphic map $\widetilde{f}:(U-E) \times K \rightarrow M$ such that $f=\widetilde{f}$ on $(U-E) \times V$.

3.2. Proof of Theorem A. By the theorem of Forelli [Ru, p. 60], there exists $r_{0}>0$ such that

$f$ is holomorphic in $\mathbb{B}_{r_{0}}^{n}$.

Put $\mathbb{B}_{*}^{n}=\mathbb{B}^{n}-\left\{z_{n}=0\right\}$. Consider the holomorphic mapping $\varphi: \mathbb{B}_{*}^{n} \rightarrow \mathbb{C}^{n}$ given by $\varphi\left(z_{1}, \ldots, z_{n}\right)=\left(z_{1} / z_{n}, \ldots, z_{n-1} / z_{n}, z_{n}\right)$. Put $\varphi\left(\mathbb{B}_{*}^{n}\right)=T$ and define $\varphi_{1}: \mathbb{B}_{*}^{n} \rightarrow T$ by $\varphi_{1}(z)=\varphi(z)$ for $z \in \mathbb{B}_{*}^{n}$. Then $\varphi_{1}$ is biholomorphic.

Put $g=f \circ \varphi_{1}^{-1}: T \rightarrow M$ and

$$
T_{R, h}=\left\{t=\left(t^{\prime}, z_{n}\right) \in T:\left\|t^{\prime}\right\|<R \text { and } 0<\left|z_{n}\right|^{2}<h /\left(1+R^{2}\right)\right\}
$$


for $R>0$ and $0<h \leq 1$. It is easy to see that $\left\{T_{R, h}\right\}$ is a family of open sets which is increasing when $h$ is increasing and $T=\bigcup\left\{T_{R, 1}: R>0\right\}=$ $\bigcup\left\{T_{R, 1}: R \in \mathbb{Q}_{+}^{*}\right\}$. From (1), it follows that $g$ is holomorphic in $T_{R, r_{0}^{2}}$ for all $R>0$.

Define

$$
\begin{array}{r}
\widetilde{\Delta}_{R}=\bar{\Delta}_{\sqrt{1 /\left(1+R^{2}\right)}}=\left\{z \in \mathbb{C}:|z| \leq \sqrt{1 /\left(1+R^{2}\right)}\right\}, \\
S_{R}=\left\{w^{\prime} \in \mathbb{B}_{R}^{n-1}: g\right. \text { does not extend to any neighbourhood } \\
\text { of } \left.\left(w^{\prime} \times \widetilde{\Delta}_{R}\right) \cap \varphi_{1}\left(\mathbb{B}_{*}^{n}\right)\right\} .
\end{array}
$$

It is easy to see that $S_{R}$ is closed. We now prove that $S_{R}$ is pluripolar. Indeed, by the hypothesis and since

$$
\frac{1}{1+\left\|w^{\prime}\right\|^{2}}>\frac{1}{1+R^{2}} \quad \text { for each } w^{\prime} \in \mathbb{B}_{R}^{n-1},
$$

it follows that the mapping $g_{w^{\prime}}\left(w_{n}\right)=g\left(w^{\prime}, w_{n}\right)=f\left(w_{n} w^{\prime}, w_{n}\right)$ is holomorphic on some neighbourhood of $\widetilde{\Delta}_{R}$. From Lemma 3.1, there exists a closed pluripolar subset $S_{R}^{\prime}$ of $\mathbb{B}_{R}^{n-1}$ such that $g$ extends to a holomorphic mapping $\left.\widetilde{g}:\left(\mathbb{B}_{R}^{n-1}-S_{R}^{\prime}\right) \times \widetilde{\Delta}_{R}\right) \rightarrow M$. Clearly, $S_{R} \subset S_{R}^{\prime}$, and hence $S_{R}$ is pluripolar.

Put $\widetilde{S}_{R}=S_{R} \times \widetilde{\Delta}_{R}$ and $\widetilde{S}=\bigcup_{R \in \mathbb{Q}_{+}^{*}} \widetilde{S}_{R}$. Clearly, $\widetilde{S}$ is a pluripolar subset of $T$.

Take any point $z=\left(z^{\prime}, z_{n}\right) \in T-\widetilde{S}$. Since $T=\bigcup_{R \in \mathbb{Q}_{+}^{*}} T_{R, 1}$, there exists $R \in \mathbb{Q}_{+}^{*}$ such that $z \in T_{R, 1}$. On the other hand, by the definition of $S_{R}$ and $\widetilde{S}_{R}$, we get $z^{\prime} \notin S_{R}$. Thus $g$ extends holomorphically over a neighbourhood of $\left(z^{\prime} \times \widetilde{\Delta}_{R}\right) \cap T$. This means that $g$ is holomorphic on an open neighbourhood of $z$. This also implies that $g$ is holomorphic on an open neighbourhood of $T-\widetilde{S}$.

Consider the mapping $p: \mathbb{C}^{n} \rightarrow \mathbb{C}^{n-1}$ given by $\left(z_{1}, \ldots, z_{n}\right) \mapsto\left(z_{1}, \ldots\right.$ $\left.\ldots, z_{n-1}\right)$ and put $T^{*}=\left\{z: z \in T\right.$ and $\left.p(z) \notin \bigcup_{R \in \mathbb{Q}_{+}^{*}} S_{R}\right\}$. Since $T^{*} \subset$ $T-\widetilde{S}$, it follows that $g$ is holomorphic on an open neighbourhood of $T^{*}$.

Since $\mathbb{B}^{n}=\bigcup_{j=1}^{n}\left(\mathbb{B}^{n}-\left\{z_{j}=0\right\}\right) \cup \mathbb{B}_{r_{0}}^{n}$, we conclude that $f$ is holomorphic on an open neighbourhood of $\mathbb{B}^{n}-\bigcup_{a \in S} l_{a}$, where $S$ is pluripolar in $\mathbb{P}^{n-1}(\mathbb{C})$.

In order to prove Theorem B we need the following lemma:

3.3. Lemma ([Shi2]). Let $M$ be a complex space having (HEP). Let $U, V$ be domains in $\mathbb{C}^{m}, \mathbb{C}^{n}$ respectively and let $V_{0}$ be an open subset of $V$. If $f: U \times V_{0} \rightarrow M$ is a holomorphic map such that $f_{z}$ extends holomorphically to $V$ for almost all $z \in U$, then $f$ extends holomorphically to $U \times V$.

3.4. Proof of Theorem B. By the theorem of Forelli [Ru, p. 60], there exists $r_{0}>0$ such that $f$ is holomorphic in $\mathbb{B}_{r_{0}}^{n}$. Put $r^{*}=\sup \{r \in(0,1)$ : $f$ is holomorphic in $\mathbb{B}_{r}^{n}$ \}. Then $f$ is holomorphic in $\mathbb{B}_{r^{*}}^{n}$. Suppose $r^{*}<1$. 
STEP 1. Take $p_{0} \in \partial \mathbb{B}_{r^{*}}^{n}$. For the point $f\left(p_{0}\right) \in M$ take $W_{0}=W_{f\left(p_{0}\right)}$, $r_{0}=r_{f\left(p_{0}\right)}, S_{0}=S_{f\left(p_{0}\right)}$ as in Definition 2.2(ii) of Hartogs type, i.e. for each $\varphi \in \operatorname{Hol}(\Delta, M)$, if $\varphi(0) \in W_{0}$ then $\varphi\left(\Delta_{r_{0}}\right) \subset S_{0}$.

Since

$$
\lim _{\alpha \rightarrow 1^{-}} \frac{r^{*}(1-\alpha)}{1-\alpha\left(r^{*}\right)^{2}}=0<r_{0},
$$

there exists $\alpha_{0} \in(0,1)$ such that

$$
\frac{r^{*}\left(1-\alpha_{0}\right)}{1-\alpha_{0}\left(r^{*}\right)^{2}}<r_{0}, \quad f\left(\alpha_{0} p_{0}\right) \in W_{0} .
$$

Since

$$
\lim _{p \rightarrow p_{0}} \frac{\|p\|\left(1-\alpha_{0}\right)}{1-\alpha_{0}\|p\|^{2}}=\frac{r^{*}\left(1-\alpha_{0}\right)}{1-\alpha_{0}\left(r^{*}\right)^{2}}<r_{0},
$$

there exists $\mathbb{B}\left(p_{0}, \delta\right) \subset \mathbb{B}^{n}$ such that $\|p\|\left(1-\alpha_{0}\right) /\left(1-\alpha_{0}\|p\|^{2}\right)<r_{0}$ for each $p \in \mathbb{B}\left(p_{0}, \delta\right)$ and

$$
f\left(\alpha_{0} \mathbb{B}\left(p_{0}, \delta\right)\right)=f\left(\mathbb{B}\left(\alpha_{0} p_{0}, \alpha_{0} \delta\right)\right) \subset W_{0} .
$$

We now prove that $f\left(\mathbb{B}\left(p_{0}, \delta\right)\right) \subset S_{0}$. Indeed, take $p \in \mathbb{B}\left(p_{0}, \delta\right)$. Consider the Möbius map $\psi: \Delta \rightarrow \Delta$ given by

$$
\psi(z)=\frac{z-\left\|\alpha_{0} p\right\|}{1-\left\|\alpha_{0} p\right\| z} .
$$

Put $\psi(\|p\|)=p^{\prime}$. Consider the map $\varphi: \Delta \rightarrow \mathbb{B}^{n}$ given by $\varphi(z)=z \cdot p /\|p\|$ and the composite map $\phi:=f \circ \varphi \circ \psi^{-1}: \Delta \rightarrow M$. Then $\phi(0)=f\left(\alpha_{0} p\right) \in W_{0}$, $\phi\left(p^{\prime}\right)=f(p)$. On the other hand, since

$$
\left|p^{\prime}\right|=\frac{\|p\|\left(1-\alpha_{0}\right)}{1-\alpha_{0}\|p\|^{2}}<r_{0},
$$

we have $p^{\prime} \in \Delta_{r_{0}}$, and hence $\phi\left(p^{\prime}\right)=f(p) \in S_{0}$.

STEP 2. We now prove that, for each $p_{0} \in \partial \mathbb{B}_{r^{*}}^{n}$, there exists $\delta_{p_{0}}>0$ such that the restriction of $f$ to $\mathbb{B}\left(p_{0}, \delta_{p_{0}}\right)$ is holomorphic. Without loss of generality we may assume that $p_{0}=\left(0, \ldots, 0, r^{*}\right)$.

By using again the mappings $\varphi_{1}, g$ and the definitions of $T, T_{R, h}$, we find that $g$ is holomorphic in $T_{R,\left(r^{*}\right)^{2}}$ for all $R>0$. By Step 1 and since $\varphi_{1}$ is biholomorphic, there exists $\delta>0$ such that $g\left(\mathbb{B}\left(p_{0}, \delta\right)\right)$ is contained in a subset $S_{0}$ of Hartogs type. Note that $\varphi_{1}\left(p_{0}\right)=p_{0}$. Since

Take a sufficiently small $\delta_{1}>0$ such that $\Delta_{\delta_{1}}^{n-1} \times \Delta\left(p_{0}, \delta_{1}\right) \subset \mathbb{B}\left(p_{0}, \delta\right)$.

$$
\lim _{\delta \rightarrow 0^{+}} \frac{\left(r^{*}\right)^{2}}{1+(n-1) \delta^{2}}=\left(r^{*}\right)^{2}>\left(r^{*}-\frac{\delta_{1}}{4}\right)^{2},
$$


there exists $\delta_{2}>0$ such that

$$
\frac{\left(r^{*}\right)^{2}}{1+(n-1) \delta_{2}^{2}}>\left(r^{*}-\frac{\delta_{1}}{4}\right)^{2}, \quad 0<\delta_{2}<\delta_{1} .
$$

This implies $\Delta_{\delta_{2}}^{n-1} \times \Delta\left(r^{*}-\delta_{1} / 2, \delta_{1} / 4\right) \subset \Delta_{\delta_{2}}^{n-1} \times \Delta\left(r^{*}, \delta_{1}\right) \subset \mathbb{B}\left(p_{0}, \delta\right)$ and $\Delta_{\delta_{2}}^{n-1} \times \Delta\left(r^{*}-\delta_{1} / 2, \delta_{1} / 4\right) \subset T_{\delta_{2},\left(r^{*}\right)^{2}}$.

By Lemma 3.3, $g$ is holomorphic in $\Delta_{\delta_{2}}^{n-1} \times \Delta\left(r^{*}, \delta_{1}\right)$. Thus the assertion of Step 2 follows from the fact that $\varphi_{1}$ is a biholomorphic mapping.

SteP 3. For each $p \in \overline{\mathbb{B}}_{r^{*}}^{*}$ put

$$
\delta_{p}=\sup \{\delta: f \text { is holomorphic in } \mathbb{B}(p, \delta)\} .
$$

By Step 2, we know that $\delta_{p}$ is positive.

On the other hand, it is easy to see that

$$
\left|\delta_{p_{0}}-\delta_{p_{1}}\right| \leq\left\|p_{0}-p_{1}\right\|, \quad \forall p_{0}, p_{1} \in \overline{\mathbb{B}}_{r^{*}} .
$$

This implies that the function $\delta: \overline{\mathbb{B}}_{r^{*}} \rightarrow \mathbb{R}_{*}^{+}$is continuous. Hence $\min _{p \in \overline{\mathbb{B}}_{r *}^{n}} \delta(p)=\delta_{r^{*}}>0$. Then $f$ is holomorphic in $\mathbb{B}_{r^{*}+\delta_{r^{*}}}^{n} \supsetneq \mathbb{B}_{r^{*}}^{n}$. This is a contradiction.

The following lemma plays an essential role in proving Theorem C:

3.5. Lemma ([T]). Let $M$ be a holomorphically convex Kähler complex space. Then $M$ has (HEP) if and only if $M$ contains no rational curves.

3.6. Proof of Theorem C. Sufficiency. This follows immediately from Theorem B.

Necessity. By Lemma 3.5, it suffices to prove that $M$ contains no rational curve.

Suppose that

(1) there exists a rational curve $\varphi: \mathbb{P}^{1}(\mathbb{C}) \rightarrow M$ and $\varphi \neq$ const.

Consider the mapping $f: \mathbb{B}^{2} \rightarrow \mathbb{P}^{1}(\mathbb{C})$ given by $(z, w) \mapsto\left[(z+w-1)^{2}\right.$ : $\left.(z-w)^{2}\right]$ for each $(z, w) \neq(1 / 2,1 / 2)$ and $f(1 / 2,1 / 2)=[1: 1]$. Then it is easy to check that $f$ is $\mathcal{C}^{\infty}$ in an open neighbourhood of the origin and the restriction of $f$ to each complex line through the origin is holomorphic. Since $M$ has (FP), $\varphi \circ f$ is holomorphic. In particular, it is continuous, and hence the following limit exists:

$$
\lim _{(z, w) \rightarrow(1 / 2,1 / 2)}(\varphi \circ f)(z, w)=a \in M .
$$

From (1), it follows that

$$
\varphi^{-1}(a) \text { is a finite set in } \mathbb{P}^{1}(\mathbb{C}) .
$$

Put $w=1 / 2+\lambda(z-1 / 2), \lambda \in \mathbb{C}$. Then

$$
\lim _{(z, w) \rightarrow(1 / 2,1 / 2)} f(z, w)=\left[(1+\lambda)^{2}:(1-\lambda)^{2}\right] .
$$


By (2), we have $\left\{\left[(1+\lambda)^{2}:(1-\lambda)^{2}\right]: \lambda \in \mathbb{C}\right\} \subset \varphi^{-1}(a)$. This contradicts $(3)$. The proof is complete.

In order to prove Theorem D we need the following lemma:

3.7. LEMMA ([Shi 3]). Let $M$ be a complex space having the meromorphic extension property. Let $U, V$ be open sets in $\mathbb{C}^{m}, \mathbb{C}^{n}$ respectively, and let $V_{0}$ be an open subset of $V$. Let $f: U \times V_{0} \rightarrow M$ be a meromorphic mapping. If $f_{z}$ has a meromorphic extension to $V$ for almost all $z \in U$, then $f$ has a meromorphic extension to $U \times V$.

3.8. Proof of Theorem $D$. We use the argument of the first part of Theorem A. By the theorem of Forelli [Ru, p. 60], there exists $r_{0}>0$ such that $g$ is holomorphic in $T_{R, r_{0}^{2}}$ for all $R>0$.

From Lemma 3.7, we deduce that $g$ is meromorphic in $T_{R, 1}$. Since $T=$ $\bigcup_{R>0} T_{R, 1}, g$ is meromorphic in $T$. On the other hand, since $\mathbb{B}^{n}=\bigcup_{i=1}^{n}\left(\mathbb{B}^{n}-\right.$ $\left.\left\{z_{i}=0\right\}\right) \cup \mathbb{B}_{r_{0}}^{n}$, we conclude that $f$ is meromorphic in $\mathbb{B}^{n}$.

3.9. Remark. The Kähler property in Theorem D cannot be omitted. Consider the Hopf surface $S=\mathbb{C}^{2}-\{0\} / z \sim 2 z$ and the canonical projection $\varphi: \mathbb{C}^{2}-\{0\} \rightarrow S$. Then $\varphi$ is holomorphic on any complex curve through 0 but does not extend meromorphically to $\mathbb{C}^{2}$. Let $f: \mathbb{B}^{2} \rightarrow S$ be the holomorphic mapping given by $f(z, w)=\varphi\left((z+w-1)^{2},(z-w)^{2}\right)$. It is easy to see that the $\operatorname{limit}_{t \rightarrow 0} \varphi(t, t)$ exists and equals $f(1 / 2,1 / 2)$. But $f$ is not meromorphic at 0 .

\section{References}

[ASY] K. Adachi, M. Suzuki and M. Yoshida, Continuation of holomorphic mappings with values in a complex Lie group, Pacific J. Math. 47 (1973), 1-4.

[Fu] H. Fujimoto, On holomorphic maps into a taut complex space, Nagoya Math. J. 46 (1972), 49-61.

[I] S. M. Ivashkovich, The Hartogs phenomenon for holomorphically convex Kähler manifolds, Math. USSR-Izv. 29 (1987), 225-232.

[JP] M. Jarnicki and P. Pflug, An extension theorem for separately holomorphic functions with pluripolar singularities, Trans. Amer. Math. Soc. 355 (2003), 1251-1267.

[Ko] S. Kobayashi, Hyperbolic Complex Spaces, Grundlehren Math. Wiss. 318, Springer, 1998.

[NZ1] Nguyen Thanh Van et A. Zeriahi, Familles de polynômes presque partout bornées, Bull. Sci. Math. 107 (1983), 81-91.

[NZ2] - - - Une extension du théorème de Hartogs sur les fonctions séparément analytiques, in: Analyse Complexe Multivariable, Récents Développements, A. Meril (ed.), EditEl, Rende, 1991, 183-194.

[Ru] W. Rudin, Function Theory in the Unit Ball of $\mathbb{C}^{n}$, Springer, 1980.

[Sha] B. Shabat, Introduction to Complex Analysis, Part II. Functions of Several Variables, Transl. Math. Monographs 110, Amer. Math. Soc., 1992. 
[Shi1] B. Shiffman, Extension of holomorphic maps into Hermitian manifolds, Math. Ann. 194 (1971), 249-258.

[Shi2] - , Hartogs theorems for separately holomorphic mappings into complex spaces, C. R. Acad. Sci. Paris Sér. I 310 (1990), 89-94.

[Shi3] -, Separately meromorphic mappings into compact Kähler manifolds, in: Contributions to Complex Analysis and Analytic Geometry, H. Skoda and J. M. Trepreau (eds.), Vieweg, Braunschweig, 1994, 243-250.

[S] J. Siciak, Separately analytic functions and envelopes of holomorphy of some lower dimensional subsets of $\mathbb{C}^{n}$, Ann. Polon. Math. 22 (1969/1970), 145-171.

[Te] T. Terada, Sur une certaine condition sous laquelle une fonction de plusieurs variables complexes est holomorphe, Publ. Res. Inst. Math. Sci. Ser. A 2 (1967), 383-396.

[T] D. D. Thai, On the $D^{*}$-extension and the Hartogs extension, Ann. Scuola Norm. Sup. Pisa 418 (1991), 13-38.

[TM] D. D. Thai and N. T. T. Mai, Hartogs-type extension theorems for separately holomorphic mappings on compact sets, Internat. J. Math. 5 (2000), 723-735.

[TP] D. D. Thai and P. N. Mai, Convergence and extension theorems in geometric function theory, Kodai Math. J. 26 (2003), 179-198.

Department of Mathematics

Hanoi University of Education

Cau Giay, Hanoi, Vietnam

E-mail: ddthai@netnam.org.vn 